\title{
Alveolar hemorrhage as the initial presentation of systemic lupus erythematosus
}

\author{
Bruna A. de Holanda, Isabela G. Menna Barreto, Isadora S. Gomes de Araujo, \\ Daniel B. de Araujo \\ Universidade Federal de Pelotas, Pelotas, Brazil
}

\begin{abstract}
Alveolar hemorrhage $(\mathrm{AH})$ is a rare syndrome that can often occur in autoimmune diseases, blood clotting disorders, infection or by acute inhalation injury, presenting rapid evolution and high mortality, especially with late diagnosis and treatment. Among the autoimmune diseases, there are reported cases in patients with primary antiphospholipid syndrome (PAPS), vasculitis and systemic lupus erythematosus (SLE). An early diagnosis is an essential tool in the successful management of this complication, requiring aggressive treatment based on vigorous immunosuppression and broad-spectrum antibiotic. We describe here a case of alveolar hemorrhage associated with glomerulonephritis as the open presentation in a patient with SLE.
\end{abstract}

Key words: systemic lupus erythematosus, respiratory insufficiency, alveolar hemorrhage, lung disease.

\section{Introduction}

Systemic lupus erythematosus (SLE) is an autoimmune disease, characterized by production of autoantibodies and for a heterogeneous clinical presentation, while most common manifestations in its onset include arthritis, cutaneousrash, photosensitivity, immune mediated cytopenias and renal involvement.

Although SLE has the potential to affect any organ, the lungs are often involved in the later stages of the disease. Pleuritis is the most common pulmonary manifestation on SLE, but there are other possible presentations, such as parenchymal disease, pulmonary vascular disease and diaphragmatic dysfunction [1]. Alveolar hemorrhage $(\mathrm{AH})$ is an unusual complication of SLE, with high mortality rate, and is extremely rare as an early manifestation of this condition [2].

\section{Case report}

A twenty-one-year-old white woman experienced initial symptoms of fever, fatigue and weight loss two months ago, evolving with malar rash, proteinuria of $2.4 \mathrm{~g}$, and the presence of macroscopic hematuria and granularcasts in urine sediment. She was admitted to our hospital for an initial evaluation, presenting positivity for ANA, anti-Sm and anti-dsDNA, and complement consumption. Two days after hospitalization she developed hemoptysis, drop in hemoglobin (from $10.4 \mathrm{~g} / \mathrm{dl}$ to $5.8 \mathrm{~g}$ / $\mathrm{dl}$ ), and hypoxemia, with chest $\mathrm{X}$-ray showing bilateral pulmonary infiltrates, requiring ICU admission, mechanical ventilation (MV) and red blood cells transfusion. A diagnosis of $\mathrm{AH}$ was made based on clinical, radiological, and laboratory findings and broad-spectrum antibiotics therapy was established associated with methylprednisolone and cyclophosphamide pulse, with a increased in hemoglobin levels $(9.8 \mathrm{~g} / \mathrm{dl})$ without any new episodes of $\mathrm{AH}$. Repeated blood cultures were negative. After ten days of the first $\mathrm{AH}$ episode, patient developed a new episode of frank hemoptysis with rapid deterioration of lung function and hemodynamic instability with marked decrease in hemoglobin $(6.2 \mathrm{~g} / \mathrm{dl})$. A chest CT scan revealed bilateral pulmonary infiltrates (Fig. 1) consistent with recurrence of $\mathrm{AH}$. 
The patient received new methylprednisolone pulse and human immunoglobulin pulses (IVIG), not being submitted to plasmapheresis due to hemodynamic instability, evolving to death after three days.

\section{Discussion}

Non-infectious pleuropulmonary involvement occurs frequently in SLE patients, affecting up to $70 \%$ of these at some point of the disease [3]. Its main clinical manifestations are presented by pleuritis with pleural effusion, lupus pneumonitis, shrinking lung syndrome, pulmonary hypertension, interstitial lung disease, pulmonary embolism, and alveolar hemorrhage $(\mathrm{AH})$. Besides SLE, other conditions that occurs with pulmonary capillaritis may also presents AH such as primary antiphospholipid syndrome, Behçets disease, Goodpasture syndrome, other vasculitides, bleeding disorders, infections, and some toxins [4]. The differential diagnosis can sometimes be difficult since some of their clinical manifestations are quite similar. Furthermore, the sudden appearance of diffuse pulmonary infiltrates in patients with SLE is a relatively rare eventre presenting a challenging diagnosis. Several pathological events might be responsible for this manifestation, including infection, congestive heart failure with acute pulmonary edema, pulmonary embolism, hemorrhage due to severe coagulopathy, and pneumonitis with or without hemorrhage [2, 5-7].

Although rare, $\mathrm{AH}$ is a severe and potentially lethal form of pulmonary involvement usually with a rapid evolution. It might occur even in the course of immunosuppressive treatment and it can present frequent recurrences [2-12]. Usually, respiratory failure, hypovolemic shock and sepsis are the main causes of death in $\mathrm{AH}$, with a mortality rate ranging from 30 to $90 \%$ [7, 9, 13, 14].

The sudden appearance of dyspnea, hypoxemia, hemoptysis, and a drop in hemoglobin levels, when associated with pulmonary interstitial or alveolar infiltrates should lead to the suspicion of $\mathrm{AH}$, especially in a patient with active disease $[2,8,12]$. Other signs and symptoms such as cough, paleness, thoracic pain, hypotension, and pulmonary crackles, may be presented as early manifestations of the condition [10, 12]. Unilateral or lobar involvement in chest X-ray was observed, although bilateral involvement is the commonest pattern [10, 12].

Alveolar hemorrhage occurs mainly in patients withprevious involvement of other organs and is frequently associated with lupus nephritis, being rare as an initial presentation of SLE $[2,8,12]$. The cornerstone in its management is a high degree of suspicion and an immediate start of immunosuppressive therapy with high doses of corticosteroid associated with cyclophosphamide. Plasmapheresis could be added to the treatment in those

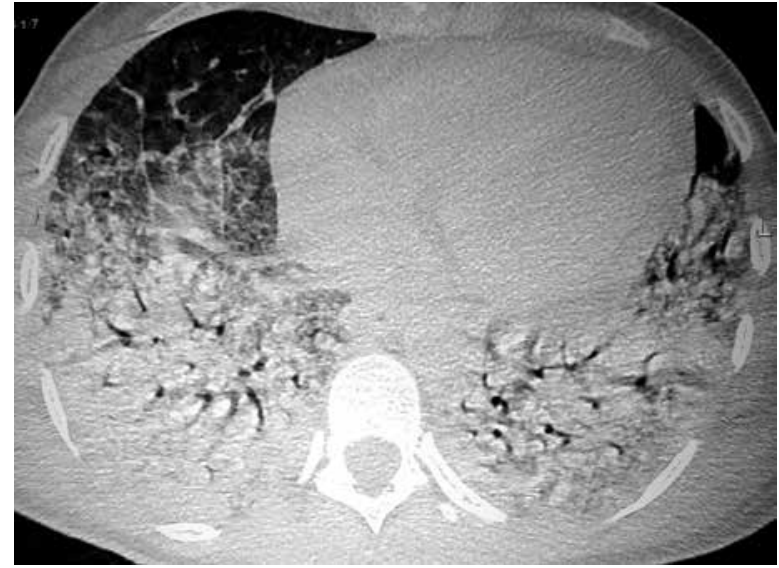

Fig. 1. Chest CT showing bilateral alveolar opacities suggestive of alveolar hemorrhage.

refractory cases $[6,8,12]$. Plasmapheresis is used in the treatment of other diseases that present $\mathrm{AH}$ including ANCA-associated vasculitis, cryoglobulinemic vasculitis, and anti-glomerular basement membrane disease, and it is believed that its action in the removal of circulating immune complexes is responsible for improvement of pulmonary capillaritis $[6,8,12]$. Other therapeutic possibilities include the use of rituximab, but we should take into account that its main mechanism of action, the depletion of CD20+ B lymphocytes, does not occur quickly jeopardizing the progress of the patient [6].

Since infections are one of the commonest causes of pulmonary symptoms in SLE patients, and besides the fact that $\mathrm{AH}$ treatment is based in immunosuppression, empiric antibiotics are mandatory until the exclusion of infection $[1,2,12,13]$. Other forms of pulmonary involvement in SLE can mimic AH, including acute lupus pneumonitis, pulmonary embolism, uremic pneumonitis, bleeding caused by clotthing disorders and pulmonary edema $[1,3,5,8,9]$.

Levels of hemoglobin, chest $X$-rays and arterial blood gas analyses are strategies that help to evaluate the evolution of this complication. Furthermore, disease activity must be monitored through complement levels, anti-dsDNA and markers of inflammation, such as C-reactive protein and erythrocyte sedimentation rate.

Although rare, the classical triad constituted by hemoptysis, sudden drop of hemoglobin levels and appearance of pulmonary infiltrates in our patient helped to establish an early diagnosis [2]. Even with a prompt diagnosis and immediate institution of antibiotics and immunosuppression, therapeutic success wasn't achieved, which demonstrates the severity of this complication $[12,13]$. 
The authors declare no conflict of interest.

\section{References}

1. Segal AM, Calabrese LH, Ahmad M, et al. The pulmonary manifestations of systemic lupus erythematosus. Semin Arthritis Rheum 1985; 14: 202-224.

2. Santos-Ocampo AS, Mandell BF. Systemic lupus erythematosus-associated alveolar hemorrhage: presentation, treatment, and outcome. I Clin Rheumatol 2000; 6: 350-355.

3. Zamora MR, Warner ML, Tuder R, et al. Diffuse alveolar emorrhage and systemic lupus erythematosus: clinical presentation, histology, survival and outcome. Medicine 1997; 76: 192-202.

4. Zandman-Goddard G. Diffuse alveolar hemorrhage in autoimmune diseases. Isr Med Assoc J 2002; 4: 461-462.

5. Carette S, Macher AM, Nussbaum A, et al. Severe, acute pulmonary disease in patients with systemic lupus erythematosus: ten years of experience at the National Institutes of Health. Semin Arthritis Rheum 1984; 14: 52-59.

6. Martínez-Martínez MU, Abud-Mendoza C. Diffuse alveolar hemorrhage in patients with systemic lupus erythematosus. Clinical manifestations, treatment, and prognosis. Reumatol Clin 2014; 10: 248-253.

7. Andrade C, Mendonça T, Farinha F, et al. Alveolar hemorrhage in systemic lupus erythematosus: a cohort review. Lupus. 2016; 25: 75-80.

8. Santos-Ocampo AS, Mandell BF, Fessler BJ. Alveolar hemorrhage in systemic lupus erythematosus - presentation and management. Chest 2000; 118: 1083-1090.

9. Schwab EP, Schumacher HR, Freundlich B, et al. Pulmonary alveolar hemorrhage in systemic lupus erythematosus. Semin Arthritis Rheum 1993; 23: 8-15.

10. Barile LA, Jara LJ, Medina-Rodriguez F, et al. Pulmonary hemorrhage in systemic lupus erythematosus. Lupus 1997; 6: 445448.

11. Koh WH, Thumboo J, Boey ML. Pulmonary hemorrhage in oriental patients with systemic lupus erythematosus. Lupus 1997; 6: 713-716.

12. Liu MF, Lee JH, Weng TH, et al. Clinical experience of 13 cases with severe pulmonary hemorrhage in systemic lupus erythematosus with active nephritis. Scand J Rheumatol 1998; 27: 291-295.

13. Araujo DB, Borba EF, Silva CA, et al. Alveolar hemorrhage: distinct features of juvenile and adult onset systemic lupus erythematosus. Lupus 2012; 21: 872-877.

14. Martinez-Martinez MU, Sturbaum AK, Alcocer-Varela J, et al. Factors associated with mortality and infections in patients with systemic lupus erythematosus with diffuse alveolar hemorrhage. J Rheumatol 2014; 41: 1656-1661. 